\title{
Numerical study on wake characteristics of high-speed trains
}

\author{
Shuan-Bao Yao · Zhen-Xu Sun · Di-Long Guo · Da-Wei Chen · Guo-Wei Yang
}

Received: 11 April 2013 / Revised: 18 July 2013 / Accepted: 6 August 2013

(C)The Chinese Society of Theoretical and Applied Mechanics and Springer-Verlag Berlin Heidelberg 2013

\begin{abstract}
Intensive turbulence exists in the wakes of high speed trains, and the aerodynamic performance of the trailing car could deteriorate rapidly due to complicated features of the vortices in the wake zone. As a result, the safety and amenity of high speed trains would face a great challenge. This paper considers mainly the mechanism of vortex formation and evolution in the train flow field. A real CRH2 model is studied, with a leading car, a middle car and a trailing car included. Different running speeds and cross wind conditions are considered, and the approaches of unsteady Reynold-averaged Navier-Stokes (URANS) and detached eddy simulation (DES) are utilized, respectively. Results reveal that DES has better capability of capturing small eddies compared to URANS. However, for large eddies, the effects of two approaches are almost the same. In conditions without cross winds, two large vortex streets stretch from the train nose and interact strongly with each other in the wake zone. With the reinforcement of the ground, a complicated wake vortex system generates and becomes strengthened as the running speed increases. However, the locations of flow separations on the train surface and the separation mechanism keep unchanged. In conditions with cross winds, three large vortices develop along the leeward side of the train, among which the weakest one has no obvious influence on the wake flow while the other two stretch to the tail of the train and combine with the helical vortices in the train wake. Thus, optimization of the aerodynamic performance of the
\end{abstract}

The project was supported by the National Key Technology R\&D Program (2009BAG12A03) and the Major State Basic Research Development Program of China (2011CB711101).

S.-B. Yao · Z.-X. Sun · D.-L. Guo (困) · D.-W. Chen · G.-W. Yang Key Laboratory for Mechanics in Fluid Solid Coupling Systems, Institute of Mechanics, CAS, 100190 Beijing, China

e-mail: jack9517@126.com

D.-W. Chen

CSR Qingdao Sifang Locomotive Co. Ltd., 266111 Qingdao, China trailing car should be aiming at reducing the intensity of the wake vortex system.

Keywords Wake flow structures · URANS - DES · High speed trains

\section{Introduction}

In recent decades, a trend towards higher running speeds has evolved in railway transportation. Sometimes, the speed may exceed $300 \mathrm{~km} / \mathrm{h}$, nearly comparable with the past airplane speeds. Due to its high length-to-diameter ratio, the aerodynamic performance of high speed trains is much more complicated compared to other ground vehicles. In high speed conditions, strong interaction between the trailing car and the ground exists and a large wake zone with intensive turbulence will generate. Eddies with different intensity generate and shed in the wake zone, which increases the inviscid drag on the trailing car and also seriously worsen the lift and side force there. Consequently, the trailing car will swing aggravatingly. As a result, the safety and amenity of passengers will also deteriorate.

With the development of high speed trains, a great deal of attention has been focussed on their aerodynamic performance. Along with the development of computer technique and new algorithms for engineering application of computational fluid dynamics (CFD), numerical simulation has been an inevitable approach to investigate aerodynamic performances of high speed trains. The flow around high speed trains is an intensive turbulent flow with a remarkable characteristic of unsteadiness [1-5]. From the viewpoint of limitation factors to the speed-up of train system, Raghunathan et al. [1] has made a thorough review on aerodynamic drag, aerodynamic noise, vibration of trains, aerodynamics of train/tunnel systems and impulse wave at the exit of tunnel. Hemmida et al. [2] has performed a detailed study on the flow field around the train with a large eddy simulation (LES) approach, analyzing the influence of the nose shape and yaw angles. Baker [3] considered the aerodynamic behavior of high speed trains with a simplified shape and de- 
scribed the nature of the flow field. Wang et al. [6] used the lattice Boltzmann method (LBM) approach to carry out a numerical investigation of the $\mathrm{CRH} 2$ high speed train at different running conditions, and typical flow structures were captured. Yang et al. [7] adopted the LES method to investigate the unsteady characteristics of high speed trains. With spectral analysis, the feature of unsteady aerodynamic forces in time domain and frequency domain has been analyzed.

In the real world, the shape of the high speed train is very complicated, with a lot of attachments, such as several bogies, pantographs and the connection parts between carriages. Sometimes, the railroad bed and tracks should also be taken into consideration. As a result, it is quite difficult to numerically simulate the flow field of high speed trains in real conditions, though the numerical approaches used in industry are rather mature. Till now, unsteady Reynold-averaged Navier-Stokes (URANS) is still the most popular approach for unsteady simulation in engineering. Due to the stringent requirement on mesh resolution, the LES approach has a lot of limitations, which is the reason for the usage of simplified model in literatures $[2,3,5,7]$. LBM is a brand new approach rapidly developed in recent years, based on a totally different theoretical frame. It utilizes a mesoscopic model to simulate the flow. However, compared to macroscopic models, the LBM approach has no obvious advantage. Moreover, further researches need to be carried out to verify its reliability and computational efficiency. The detached eddy simulation (DES) [8] approach originates from solving large separation problems with high Reynolds numbers. The basic idea of DES is to use the RANS approach to simulate the flow in near-wall regions and to use the LES approach to simulate the large detached eddies in regions away from the walls. This approach benefits from advantages of both URANS and LES approaches and the required amount of mesh is just between those of LES and URANS. In the present work, both URANS and DES are utilized to study the nature of wake flow around a prototype of the $\mathrm{CRH} 2$ high speed train at different running speeds and cross wind conditions. A comparative study has been performed between the results from URANS and those from DES. Computational results could shed lights on the aerodynamic optimization with an aim to increase the safety and amenity of trailing cars.

\section{Computational models}

A real model of $\mathrm{CRH} 2$ high speed trains is adopted in this paper, with a locomotive, a middle car and a trailing car included. Considering that the attached parts (pantographs, cab roof vanes) have nearly no effect on the wake flow, these attachments are excluded from the simulation. However, the connection parts between the carriages and the bogies are included due to the intensive turbulence in these regions, which could exert a great impact on the evolution of vortices in the wake zone. Figure 1 shows the computational model used in the present work. The length of leading car is $25.7 \mathrm{~m}$, the geometry of trailing car is the same as the leading car, and the length of middle car is $25 \mathrm{~m}$, so the total length of the model is $76.4 \mathrm{~m}$.

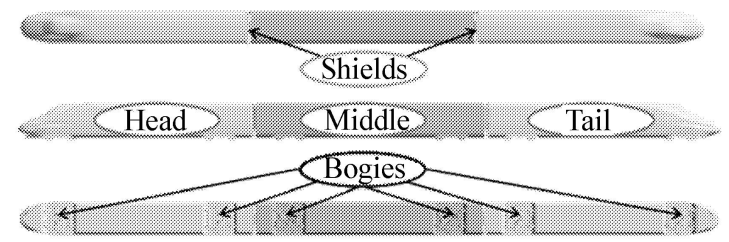

Fig. 1 The CRH2 model

\section{Computational algorithms}

Nowadays, the most widely used numerical approaches to deal with complicated engineering problems are still RANS and URANS methods. Meanwhile, the hybrid approaches based on RANS/LES have also been adopted to solve some engineering problems [9]. Due to the characteristics of flow field around high speed trains, tens of millions of grids should be ensured for actual models of high speed trains. Considering the limitation in computational time and the amount of grids, the URANS approach has been adopted to simulate the wake flow under different running speeds conditions. Meanwhile, the DES approach is utilized to deal with one of the above cases for a comparative study. In conditions with cross winds, the wake flow and the flow field in the leeward side of the train become more intensive in turbulence, and a large scale separation flow generates. Consequently, the DES approach is chosen to precisely capture typical vortex structures in these zones.

\subsection{URANS}

The concept of URANS is based on the triple decomposition of time-dependent variables, where each instantaneous variable, such as velocity and pressure, is decomposed into longtime averaged, periodic, and turbulent components $[10,11]$. Typical RANS equations are solved in an unsteady way, even if the boundary conditions are steady [9]. The fluctuating component results in the Reynolds stress terms in the RANS equations, which need to be enclosed by turbulence models. However, there is no universal model to solve all turbulence flow problems. In the present work, the flow Mach number ranges from 0.245 to 0.327 , indicating that the flow can be treated as either compressible or incompressible flows. Considering the unsteadiness in specific locations of the flow field, the $k-\omega$ SST model is adopted for turbulence enclosure. It is a kind of hybrid model which turns to the Wilcox $k-\omega$ model in near-wall zones while turns to the $k-\varepsilon$ model outside the boundary layers. A hybrid function is utilized for the transition of models. Essentially, it belongs to a two-equation eddy viscosity model with its transport variables integrated to the walls for incompressible/compressible flows [12]. 


\subsection{DES}

The DES approach is a hybrid method which combines RANS and LES together, and benefits a lot in solving large separation problems with high Reynolds numbers $[8,9,13,14]$. DES usually keeps high accuracy in predicting aerodynamic drag and separation locations for large separation flows around ground transport vehicles $[15,16]$. With the help of DES, the complicated trailing vortices in the wake zone could be precisely captured. The most popular DES approaches could be divided into two kinds: one is based on the SA model and the other is based on the SST turbulence model, which is used in the present paper. For the latter one, it is realized by replacing the turbulence length scale factor with the function $\min \left[n, 0.65 \Delta_{\max }\right]$, in which: $\Delta_{\max }=\max [\Delta x, \Delta y, \Delta z]$, where $\Delta x, \Delta y, \Delta z$ are the length of the side of the controlling volume respectively. As a result, the transition between URANS and LES could be achieved [10].

\subsection{Discritization methods and boundary conditions}

In this paper, all the computation results presented are obtained with CD-Adapco's codes Star CCM+ 4.04. The finite volume method based on cells is adopted for the discritization of the controlling equations. A second order upwind scheme is used for convection terms, while for viscous terms the second order central differentiation scheme is adopted. A completely implicit scheme lower-upper symmetric GaussSeidel (LU-SGS) methods is adopted for time discritization. In Ref. [3], Baker pointed out that the helical vortices in the train wake underwent some sort of regular oscillation for a Strouhal number of around 0.11-0.14, so the physics time step used in the simulation is set to be $0.5 \mathrm{~ms}$ to exactly capture the formation and evolution of vortices in every period. Besides, the inner steps are chosen as 10 and the CourantFriedrichs-Lewy (CFL) number is 5. Meanwhile, the standard wall function [17] is used for near wall treatment. Considering that the high speed train runs at a subsonic speed, the Riemann invariant is adopted to solve the variables at far field boundaries. As a result, the inlet, outlet and far field of the computational domain are all set to have non-reflective boundary conditions. A no-slip wall condition is used on the train surface. In order to simulate the ground effect caused by the relative motion between the train and the ground, a moving wall boundary with the same speed as the inlet flow is adopted for the ground.

\subsection{Cases summation}

The generation and evolution of trailing vortices in the wake zone are seriously affected by the running speed and cross wind conditions. In the present work, several running speeds and cross wind conditions are considered, as listed in Table 1.

\subsection{Computational domain and mesh}

Taking $H$, the height of the high speed train, as the characteristic length, the upstream length is set as $22 \mathrm{H}$ while the downstream length of the domain is set as $44 H$. The width and height of the domain are set as $8.73 \mathrm{H}$ and $11.57 \mathrm{H}$, respectively, the distance between the ground and the bottom of the train body is $0.0513 H$, just as Fig. 2 shows.

Table 1 Cases summation

\begin{tabular}{lll}
\hline & Running speed $/\left(\mathrm{km} \cdot \mathrm{h}^{-1}\right)$ & Cross wind speed $/\left(\mathrm{m} \cdot \mathrm{s}^{-1}\right)$ \\
\hline Case 1 & 300 & 0 \\
Case 2 & 350 & 0 \\
Case 3 & 400 & 0 \\
Case 4 & 300 & 20 \\
\hline
\end{tabular}
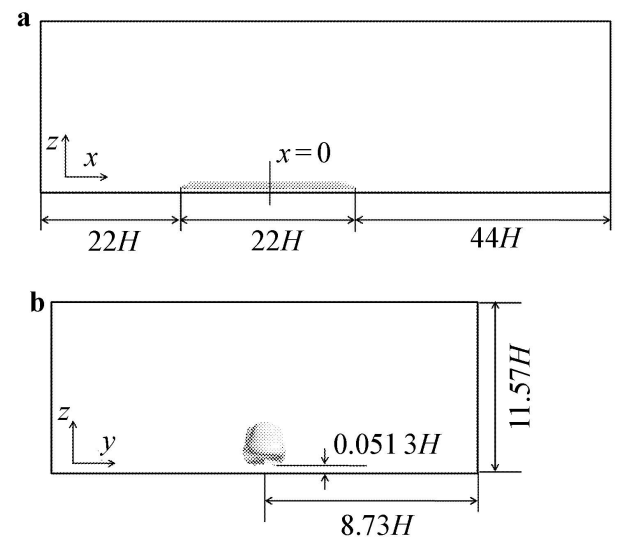

Fig. 2 Computational domain

The trimmer mesh has been utilized in the present work and the prism layers are also adopted in the near wall zone. The boundary layer thickness of the train surface is very large due to the real CRH2 model and high length-todiameter ratio. Baker [3] analyzed different distributions of boundary layer around varieties of train models, the results show that there is little growth of boundary layer thickness along the full scale train model when the flow reaches trailing car, and the value is about $1.2 \mathrm{~m}$. Besides, there is little influence of train model on the boundary layer thickness. For such a boundary layer distribution, if the calculation is carried out up to the wall, the amount of meshes is unbearable. Thus, the standard wall function method [17] is adopted in this paper, then a coarse mesh can be used to improve the computational efficiency. The $y^{+}$value is controlled in 30120 in order to meet the requirement of the wall function method. The values of $x^{+}$and $z^{+}$are controlled by the minimum size of volume mesh. All the cells of the trimmer mesh are hexahedrons. Therefore, the $x^{+}$value is equal to the $z^{+}$ value, which is about 50 times larger than the $y^{+}$value. The size of the vortex structures around high-speed trains usually varies greatly. Hemida et al. [2] investigated the flow structure of a simplified high speed train in a cross wind condition and noted that the length of the most large vortex on the leeward side is 6 times of the train height. The aerodynamic forces of trailing car is controlled by the large-scale eddies rather than the small-scale eddies far away from the trailing 
zone [3]. If the eddies of all scales is simulated, the amount of required meshes is unbearable. Therefore, considering the size of large-scale vortexes, the mesh in the wake flow is densified and the minimum volume mesh size is $0.017 \mathrm{H}$ so as to control the values of $x^{+}$and $z^{+}$. Since flow separation is usually occurs in the dramatically changing zones, for these areas, the mesh is densified adaptively during the mesh generation, so we do not consider the mesh resolution near the separation zones. The total number of volume is about 28 millions, and the representative meshes at different locations are shown in Fig. 3. Overall distribution of grids and local grids are shown in Fig. 3. It is difficult to find suitable experimental results to validate the reasonableness of the mesh layout methods adopted in this paper, so we will analyze the CFD methods and mesh resolution through a simplified wind tunnel experiment in the next section.

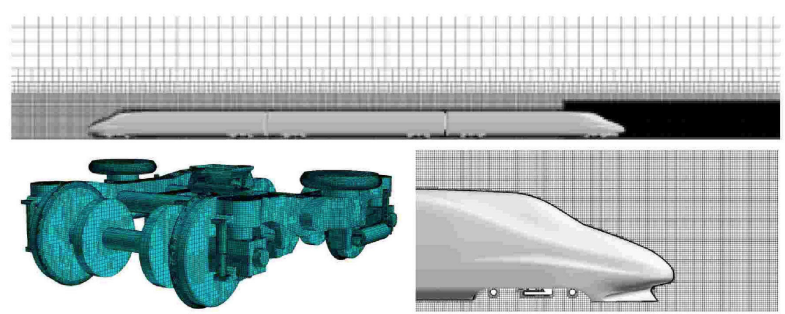

Fig. 3 Representative mesh at different locations

\subsection{CFD validation}

Schulte Werning et al. [18] investigated the vortex structure in a simplified train wake through the use of URANS methods, explained the mechanism of formation, development and evolution of helical vortex clearly, and got the shedding frequency of wake vortex. Mikael et al. [19] investigated the flow flied around a bogie under the high-speed train through CFD methods with STAR-CD and STAR-CCM+. The results show that DES may get a better simulation result of large separation than RANS, however, considering the computational efficiency, RANS is still the first choice to solve practical engineering problems. A ground transport model experiment has been done by Storms et al. [20] in NASA Ames $(2113.6 \times 3048) \mathrm{mm}^{2}$ wind tunnel, and the numerical methods and the reasonableness of meshes layout used in this paper will be validated by this wind tunnel experiment. The experiment model is shown in Fig. 4. The inlet velocity is $90.26 \mathrm{~m} / \mathrm{s}$, and the Reynolds number that based on inlet velocity and width of the model is $2 \times 10^{6}$. The mesh discretization is the same as the method introduced in Sect. 3.5. Considering the smaller size of the model, the mesh size of the densified zone is smaller, of which the minimum mesh size is $1 \mathrm{~cm}$, and the total number of volume meshes is about 8.5 millions.
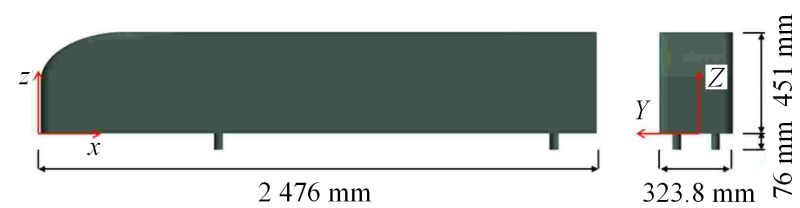

Fig. 4 The model of wind tunnel

Figure 5 shows the pressure distribution on different sections of the model. The horizontal axis coordinates are nondimensionalized by the width of the model. The formula of $C p$ is the same as adopted in Ref. [20]. The results show that the mesh is fine enough to simulate small-scale vortex structures, and for the $C p$ distribution on the front and middle surface of the model, both methods are in good agreement with the experimental values, they give almost the same results. However, DES can better simulate the flow separation occurring in the wake of the model, and obtain more accurate $C p$ distribution on the corners of the trailing surface, which works better than URANS. Although URANS does not do as well as DES for large separation flow simulation, considering mesh resolution for complex geometry and computation time, URANS is still a favorable CFD method to solve practical engineering problems with a large amount of mesh.
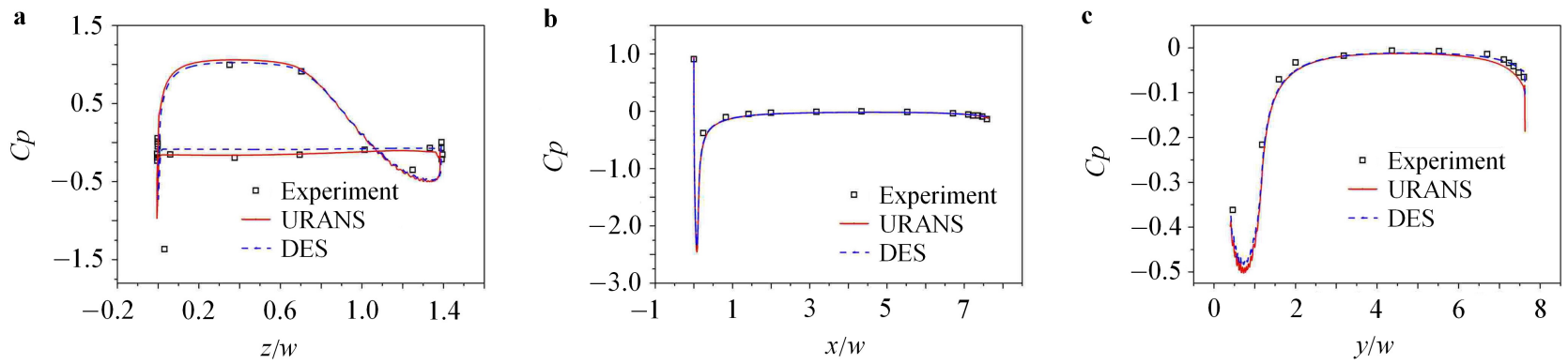

Fig. 5 a The $C p$ distribution on longitudinal section; b The $C p$ distribution on side section; $\mathbf{c}$ The $C p$ distribution on upper section $(w=323.8 \mathrm{~mm})$

\section{Results and discussions}

\subsection{Comparison of URANS and DES results}

In order to perform a comparative study between the results of URANS and DES, the mesh independency should be ensured. In this paper, the same mesh is adopted for both approaches. In the simulation, the train is assumed to run at a speed of $300 \mathrm{~km} / \mathrm{h}$ and no cross wind is considered. 
The aerodynamic performance of the trailing car is severely affected by the generation, evolution and collapse of the vortices in the wake zone. In conditions without cross wind, the lift and side force are the key aerodynamic forces that influence the safety and amenity of the trailing car. As Fig. 6 shows, the oscillation period and amplitude of the lift coefficient $\mathrm{Cl}$ are obviously larger than those of the side force coefficient $C s$. A positive value of lift can be observed which would decrease the contact between the wheels and the track. As the lift grows bigger, train derailment may take place. The side force is oscillating around 0 , which are mainly a re-

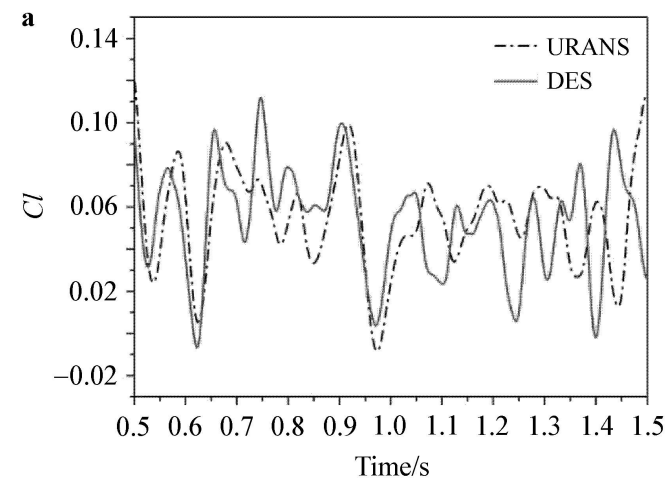

Fig. 6 Time histories of the $C l$ and $C s$ of the trail car

Figure 7 shows the instantaneous iso-surface of the second invariant of the velocity gradient $Q$ in the wake flow. It can be obviously observed that strong interaction exists between the large vortices and small vortices, which makes the wake flow much more complicated. Both DES and URANS can precisely capture the large vortices 1,2 , and 3 , as shown in Fig. 7. The locations where eddies sheds from are almost the same for the two approaches. However, small eddies could be better captured by DES than URANS. For the wake flow with large separations, results from DES are much closer to the reality than those from URANS.

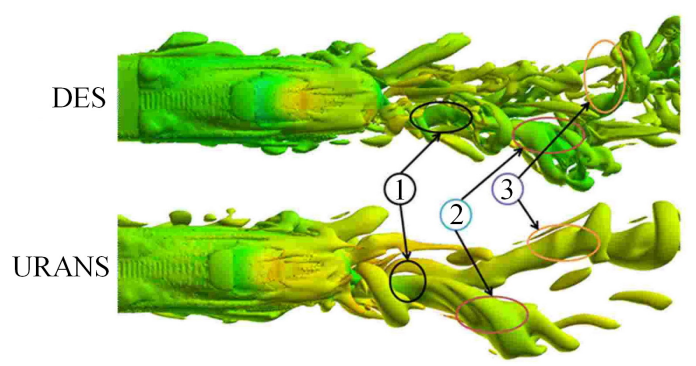

Fig. 7 An iso-surface of the second invariant of the velocity gradient, $Q=100, t=1.5 \mathrm{~s}$

Figure 8 shows the vorticity contour on different sections in the $z$-direction. It can be observed that the vorticity distribution around the bottom of the train and the wake zone is almost the same for the two methods. In regions away from the nose of the trailing car, only two strong vortex sult of spatial asymmetry of the three-dimensional flow field in the wake. Reasonable agreement of the two approaches could be observed. As time grows, the wake flow turns to fully developed turbulent flow. Due to the different capacities of capturing small eddies, the instantaneous aerodynamic results vary a lot but the mean values remain the same. Results indicate that the two methods can both capture the large eddies which have bigger effects on the aerodynamic forces of the trailing car. However, big difference exists in the captured pictures of small eddies.

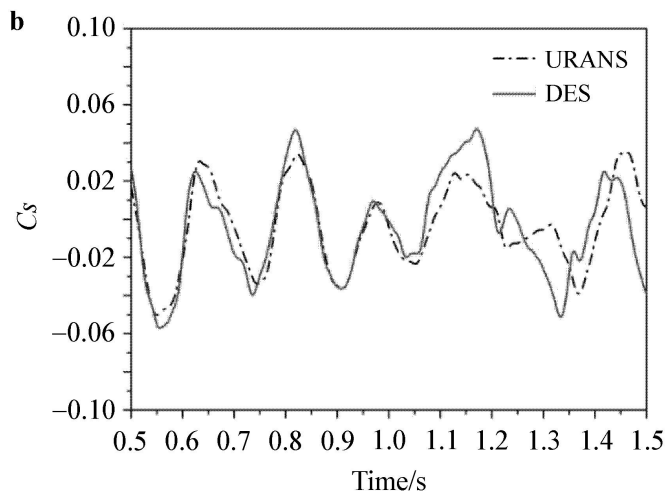

structures could be detected from URANS while more small eddies could be observed from DES. At $H=2 \mathrm{~m}$, URANS could capture vortex structures with large cores yet low intensity while DES could obtain vortex structures with small cores yet high intensity.

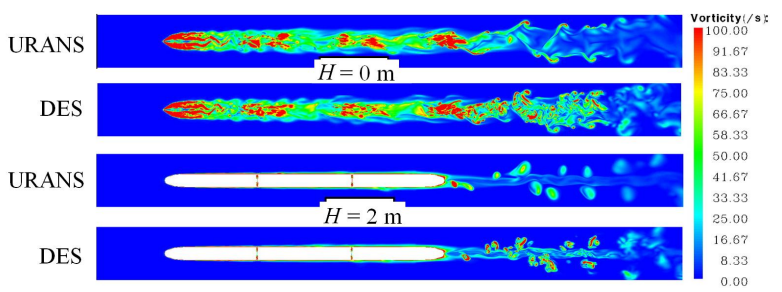

Fig. 8 Vortices contour on different sections in the $z$-direction

As Fig. 9 shows, the vorticity and velocity contour at $y=0 \mathrm{~m}$ vary a lot for the two approaches. URANS has a poor ability to capture the vortex structures since some small eddies which interact strongly with the ground could not been obtained. However, the two approaches still have something in common. For example, the initial vortices generated from the interaction between viscosity and velocity shears are in good agreement. Besides, the disturbed height of the trailing wake zone predicted by DES and URANS is also the same.

Although results from URANS are not as good as those from DES, the basic vortex structures in the wake zone could be obtained and the locations where large eddies sheds from could be captured too. Considering that DES is much stricter 
in mesh resolution and computational time than URANS, the following analyses on evolution of trailing vortices at different running speeds are based on the URANS approach. However, DES will be used for cross wind study since more complicated flow field exists in this condition.

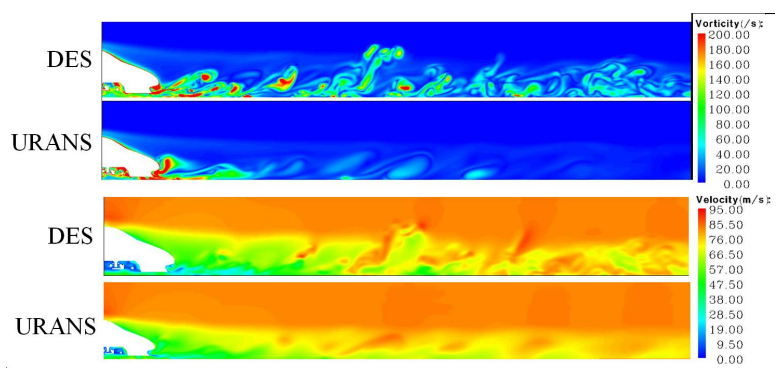

Fig. 9 Vortices and velocity contour on the $y=0$ section

4.2 Evolution of trailing vortices at different running speeds

When a high speed train is running, the air around the train also moves due to the viscosity effect, resulting in a highly turbulent wake flow. In order to better exhibit the trailing vortex structures and the evolution process of the trailing vortices, the pressure and vortices contour are mainly discussed in this section.

Pressure contour and iso-surface of $Q$ at different running speeds are shown in Fig. 10. It can be observed that two big intensive cores detach from the nose of the trailing car and develop into a helical mode in the wake zone. Strong interactions occur between the helical vortices and the ground, resulting in smaller eddies. These small eddies continue to develop in helical mode with their cores growing bigger and bigger. Meanwhile, interactions between the small eddies and the ground still exist. As a result, the vertex structures in the wake zone become much more complicated.

$\mathbf{a}$

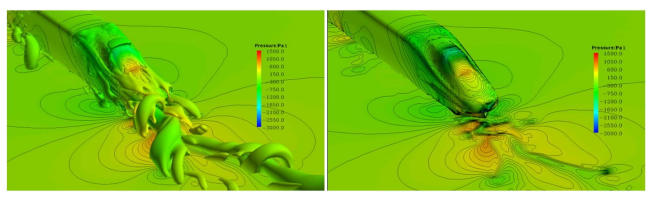

b
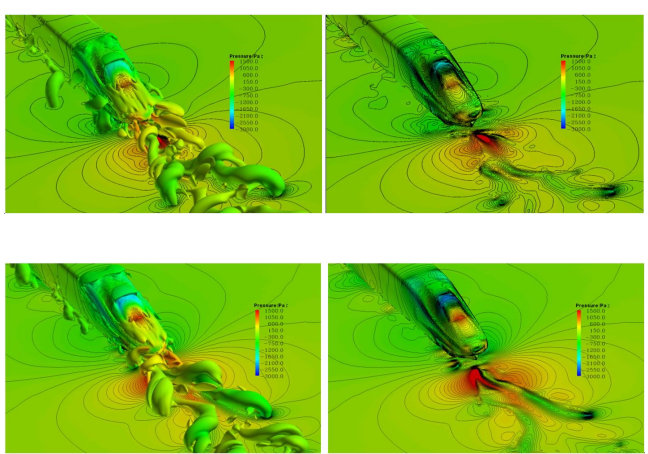

Fig. 10 Instantaneous pressure contour and iso-surface of $Q$ at different speeds $(Q=100, t=1.5 \mathrm{~s})$. a $V=300 \mathrm{~km} / \mathrm{h} ; \mathbf{b} V=350 \mathrm{~km} / \mathrm{h}$; c $V=400 \mathrm{~km} / \mathrm{h}$
As the running speed increases, the intensity of the high pressure zone just beneath the trailing nose becomes stronger, and the range of the high pressure zone becomes larger too. The vortex structures in the wake zone vary obviously at different running speeds. When the train runs at the speed of $300 \mathrm{~km} / \mathrm{h}$, the cores of the two helical vortices are very large and keep at a relatively long distance before collapse. When the speed reaches $400 \mathrm{~km} / \mathrm{h}$, the helical vortices detach from the trailing nose with high intensity, and collapse quickly as soon as they interact with the ground. Smaller eddies then generate due to the collapse and propagate in a shorter period, resulting in stronger interaction with the ground. Consequently, the wake flow at a speed of $400 \mathrm{~km} / \mathrm{h}$ is more intensive turbulent than that at a speed of $300 \mathrm{~km} / \mathrm{h}$.

Surface streamlines on the trailing car at different running speeds are shown in Fig. 11. As seen in Fig. 11, the separation lines locate symmetrically on the surface of the trailing car. Two separation line can be observed on each side of the streamlined surface. S1 and S2 on the one side join together with the other two separation lines on the other side at the tip of the trailing nose. The downstream airflow could be divided into three kinds: the flow just on top of the streamlined surface, the flow on the lateral surfaces and the flow beneath the surface. The velocity decreases rapidly as the flow on top of the streamlined surface goes downward, while the velocity of the flow on the side surfaces remains unchanged, resulting in a strong velocity shear layer. As the velocity gradient grows bigger, shear layer separations could take place. However, the locations of separation lines remain the same no matter how high the running speed is, as shown in Fig. 11, indicating that the locations where trailing vortices detach from have no relation with the running speed.
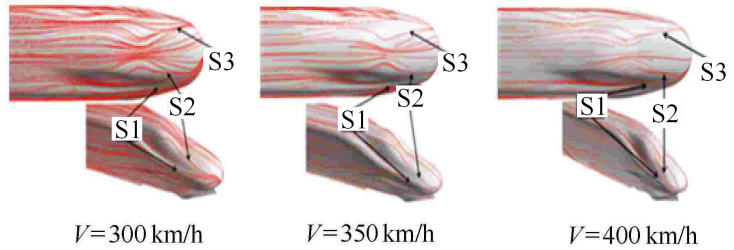

Fig. 11 Surface streamlines on the trail car at different speeds

Figure 12 shows the instantaneous pressure contour on the ground at $t=1.5 \mathrm{~s}$ at different speeds. The largest pressure gradient could be observed in the region around the leading nose. Meanwhile, the region around the last bogie and the trailing nose has also a relatively large pressure gradient. In the place between the locomotive and the trailing car, the pressure keeps more or less constant. In the wake zone, due to the shedding of trailing vortices, intermittent high pressure zone could be observed. As the velocity increases, the pressure gradient grows larger accordingly, especially in the zone around the leading nose and the trailing nose. The zone around the train is disturbed proportionally 


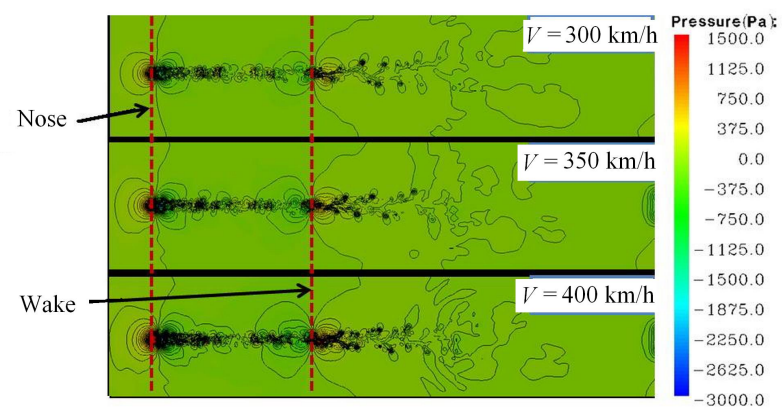

Fig. 12 Instantaneous pressure contour on the ground $(t=1.5 \mathrm{~s})$

in response to the changes of running speeds. The higher the running speed, the wider the disturbed zone.

Figure 13 shows the instantaneous velocity and vortices contour on different sections in the $z$-direction. In the section near the ground, two flows with low velocity generate around the first bogie and propagate downward until they join together. Vortices with different intensity then arise from the interaction among the above flow and those from other bogies and inter-spacing parts. When reaching the wake zone, the low-speed flow interacts with the trailing vortices, and vortex street structures could be observed. The intensity and the distance between adjacent vortex cores change with the running speed. The vortex streets become much more intensive in turbulence as the speed grows higher. In the zone away from the ground, $z=2 \mathrm{~m}$ for instance, the flow around the train turns stable and the intensity of the vortex cores turns weaker. Meanwhile, the wake zone becomes gradually out of the influence of the ground. In the even higher zone, $z=3 \mathrm{~m}$, the disturbance due to the train vanishes, and hardly any trailing vortices could be observed. In conclusion, the generation of complicated wake flow is closely related to the bottom accessories of the train and the ground effect. As the speed increases, the ground effect on the wake flow is more obvious and the cores of the trailing vortices become much higher.

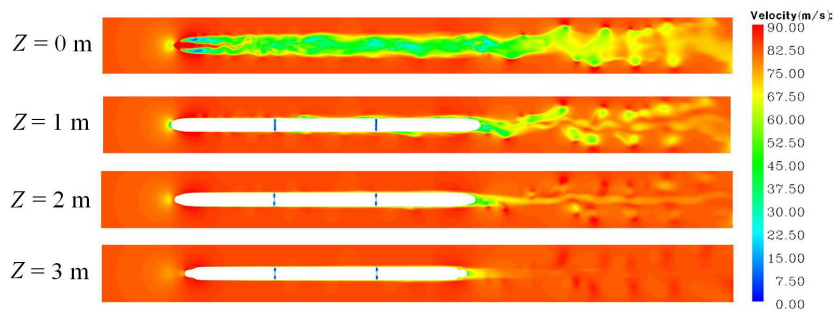

b

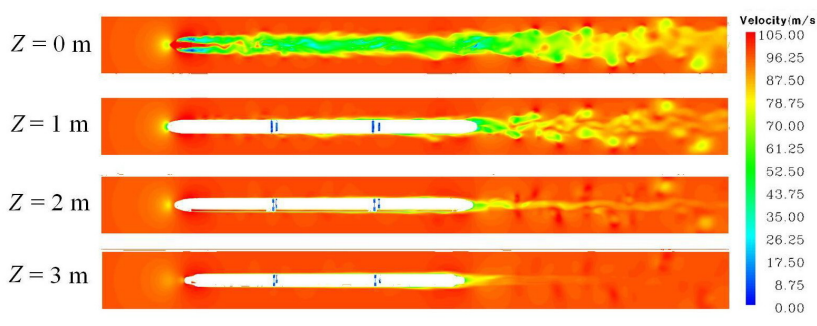

c

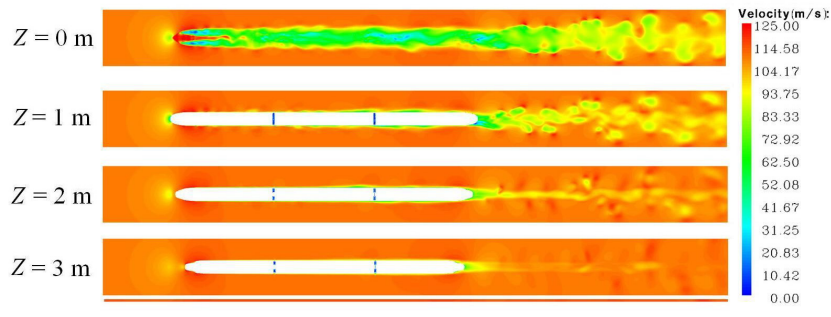

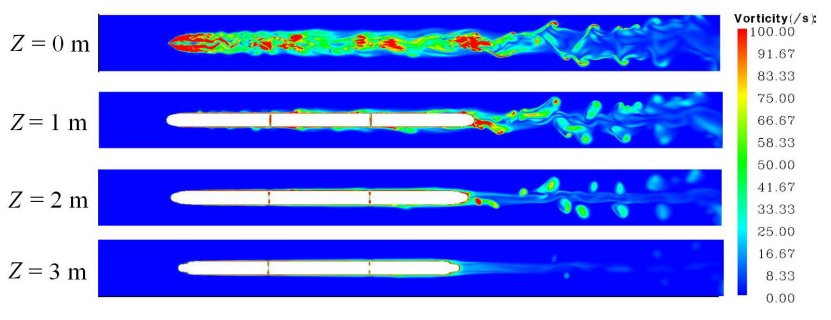
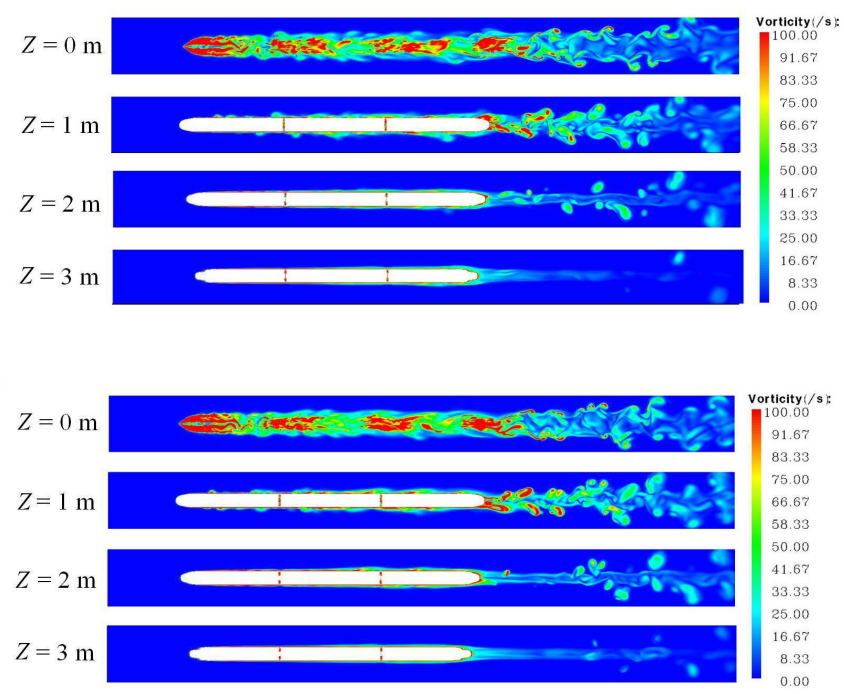

Fig. 13 Instantaneous velocity and vortictiy contour on different sections in the $z$-direction $(t=1.5 \mathrm{~s})$. a $V=300 \mathrm{~km} / \mathrm{h} ; \mathbf{b} V=350 \mathrm{~km} / \mathrm{h}$; c $V=400 \mathrm{~km} / \mathrm{h}$

Figure 14a shows the instantaneous vorticity contour on different cross sections when the running speed is $300 \mathrm{~km} / \mathrm{h}$. Two vortices with different intensity originate at the corners between the lateral sides of the train and the ground. The vortex intensity varies a lot as the two vortices go through the disturbance of the last bogie. In the zone just downward of the trailing nose, where $x=39 \mathrm{~m}$, these two vortices couple together. However, they decouple at $x=42 \mathrm{~m}$ again, however, they still interact strongly with each other. The vortex cores keep very large until $x=65 \mathrm{~m}$, even though the vor- 
tex intensity is very weak. During the propagation of the two vortices, the stronger one is always on top of the weaker one. Figure 14b shows the evolution of trailing vortices in the wake zone at different speeds. As can be seen, different running speeds lead to different propagation process of the trailing vortices. Besides, the locations where the two vortices appear alternatively and the locations where large vortices collapse are different too. As the velocity grows higher, lots of smaller and stronger eddies will generate around the trailing nose, and stronger interaction among these eddies could be observed in the downstream. Figure $14 \mathrm{c}$ shows the vorticity contour of the longitudinal section at the speed of $300 \mathrm{~km} / \mathrm{h}$. It can be observed that the height of the disturbed zone in the wake is a little lower than the height of the train

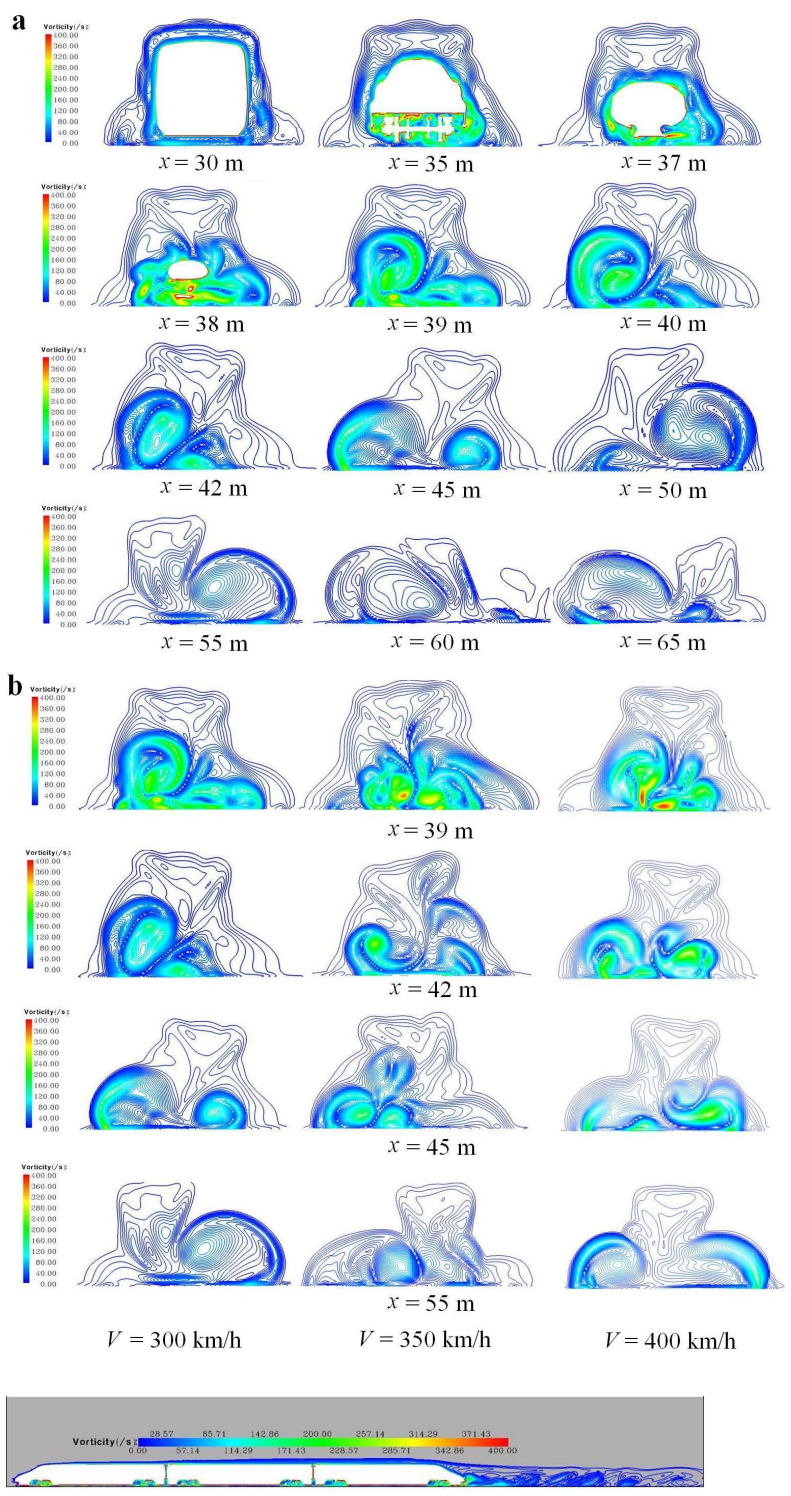

Fig. 14 a Vorticity contour on different cross sections at $V=$ $300 \mathrm{~km} / \mathrm{h}(t=1.5 \mathrm{~s}) ; \mathbf{b}$ Vorticity contour on specific cross sections at different velocities $(t=1.5 \mathrm{~s})$; $\mathbf{c}$ Vorticity contour in the longitudinal section at $V=300 \mathrm{~km} / \mathrm{h}(t=1.5 \mathrm{~s})$ and keeps essentially unchanged during the propagation of the trailing vortices. However the vortex intensity grows weaker during the propagation.

Figure 15 shows the position of separation lines at different instants on the upper surface of the trailing car at $400 \mathrm{~km} / \mathrm{h}$. The separation lines S2 and S3 swing a lot when time grows, and the position of $\mathrm{S} 2$ and $\mathrm{S} 3$ are not symmetrically distributed along the axis of the train running direction ( $x$-axis). At $t=1.1 \mathrm{~s}, \theta_{1}$ is greater than $\theta_{2}$, while at $t=1.15 \mathrm{~s}, \mathrm{~S} 2$ is closer to the $x$-axis, S3 is far away from the $x$-axis. At $t=1.2 \mathrm{~s}$, the position of $\mathrm{S} 2$ is close to the location at $t=1.15 \mathrm{~s}$, while S3 is starting to be close to the $x$-axis. At $t=1.25 \mathrm{~s}, \mathrm{~S} 2$ gradually moves in a direction away from the $x$-axis, and $\mathrm{S} 3$ is still closing to the $x$-axis. At $t=1.3 \mathrm{~s}$, $\mathrm{S} 2$ is still away from the $x$-axis, S3 begins to move in a direction away from the $x$-axis too. Through position changes of S2 and S3 when time changes, we can know that the two large vortex shedding from the upper surface of the tailing car shows obviously unsteady characteristics, which is the main factor to excite oscillations of the trailing car around the longitudinal axis.
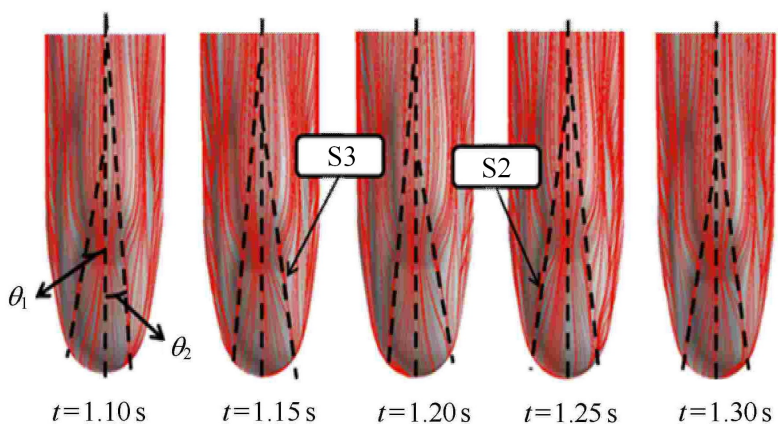

Fig. 15 The position of separation lines at different times at the speed of $400 \mathrm{~km} / \mathrm{h}$

Figure 16 shows the time histories of $C l$ and $C s$ of the trailing car at the speed of $400 \mathrm{~km} / \mathrm{h}$. As can be seen, the values of the force change a lot at different times. Compared to $C s$, the fluctuation of $C l$ is more serious. From $t=1.1 \mathrm{~s}$ to $t=1.3 \mathrm{~s}$, there are three peaks and three valleys appearing in $\mathrm{Cl}$, while only one peak and two valleys appear in $C s$. From Fig. 15 we can know that the position of S2 and S3 have two big jumps during this period, one is at $t=1.1 \mathrm{~s}$ to $t=1.15 \mathrm{~s}$, and the other one is at $t=1.2 \mathrm{~s}$ to $t=1.25 \mathrm{~s}$. These two jumps are consistent with the fluctuations of $C s$. At $t=1.1 \mathrm{~s}$, the $C s$ is at the valley position, while at $t=1.15 \mathrm{~s}$, the $C s$ is at the peak position, then at $t=1.25 \mathrm{~s}$, the $C s$ is at the valley position again. Fluctuation of $\mathrm{Cl}$ is not apparently consistent with the oscillations of S2 and S3. It can be speculated that the side force of the trailing car is dominated by large eddies detaching from the trailing cone, but the lift force of the trailing car is caused by the interaction of large eddies and small eddies near the trailing cone. 


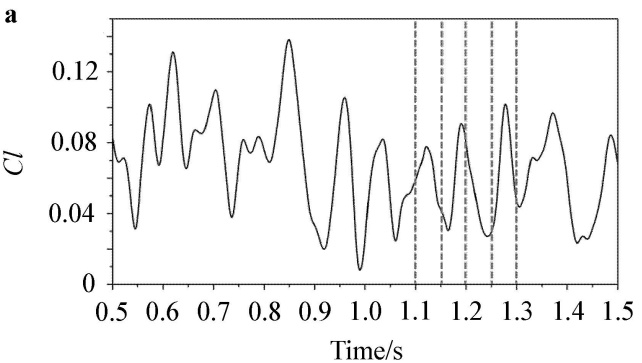

b

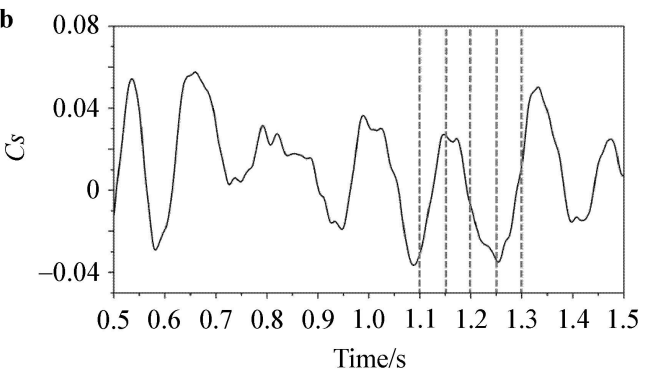

Fig. 16 The time histories of $C l$ and $C s$ of the trailing car at the speed of $400 \mathrm{~km} / \mathrm{h}$

Figure 17 shows the power spectral density of $\mathrm{Cl}$ and $C s$ at the speed of $400 \mathrm{~km} / \mathrm{h}$. The dominant frequency of $C l$ and $C s$ are both $8 \mathrm{~Hz}$. However, the number of secondary frequency of $C l$ is obviously much more than that of $C s$. Thus, it can be learned that the fluctuation of $C l$ and $C s$ is mainly caused by alternately moving of two large vortices detaching from the upper surface of the trailing car. The small eddies in near wake flow field have little effect on $C s$, but have a great influence on $\mathrm{Cl}$.
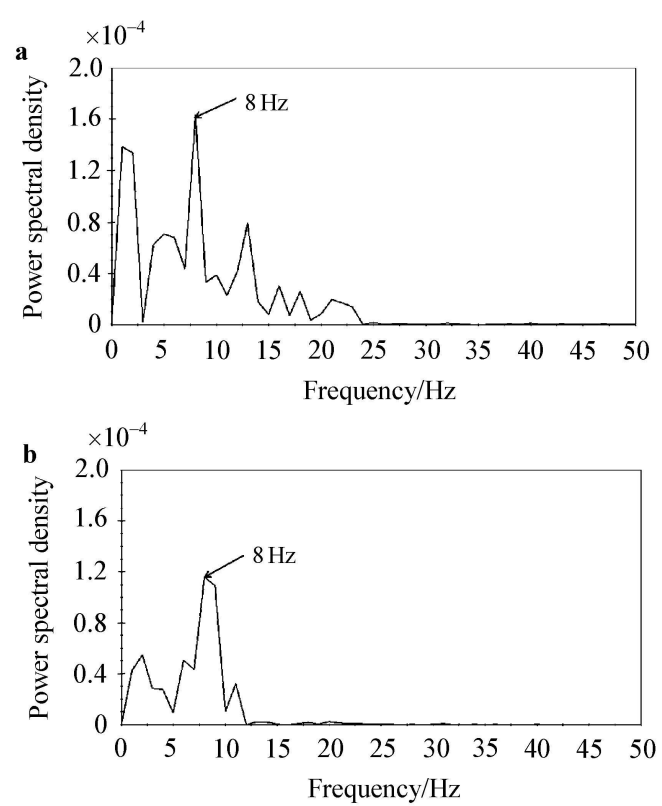

Fig. 17 The power spectral density of $\mathbf{a} C l$ and $\mathbf{b} C s$ at the speed of $400 \mathrm{~km} / \mathrm{h}$
4.3 Evolution of trailing vortices in cross wind conditions

In order to capture the small vortices on the leeward side of the train, the mesh is redistributed in this case. The zone on the leeward side is densified, while the former upwind densified zone turns a little smaller. The smallest size of the cell is $0.017 \mathrm{H}$ and the total amount of the domain is around 30.7 millions.

In cross wind conditions, the vortices on the leeward side and the complicated wake zone will result in worse aerodynamic lift force, side force and overturning moment, which would pose a severe challenge to the safety of the train. Considering that the flow field calculated from simplified model has a big difference with that around the real model, the prototype of $\mathrm{CRH} 2$ train with three carriages has been adopted and DES is utilized for the simulation.

Compared to the flow field in conditions without cross wind, significant variation can be observed in cross wind conditions. Figure 18 shows the streamlines on different cross sections at $t=1.5 \mathrm{~s}$. It can be seen that two large vortices V1 and V2 generate on the leeward side. As propagating to the trail wake, $\mathrm{V} 1$ keeps the same size while $\mathrm{V} 2$ grows smaller and smaller. A new vortex V3 generates at $x=39 \mathrm{~m}$, which is just downward of the trail nose. In the meantime, a smaller vortex V4 generates just beneath V3, which is a result of flow separations around the cowcatcher. V3 splits into two smaller eddies at $x=40 \mathrm{~m}$. As the flow propagates farther, at $x=45 \mathrm{~m}, \mathrm{~V} 1$ gets obviously smaller, V2 disappears and the three smaller eddies recombine together to form a bigger vortex. At $x=55 \mathrm{~m}$, the above two vortices still exist but the vortex intensity become rather weak.

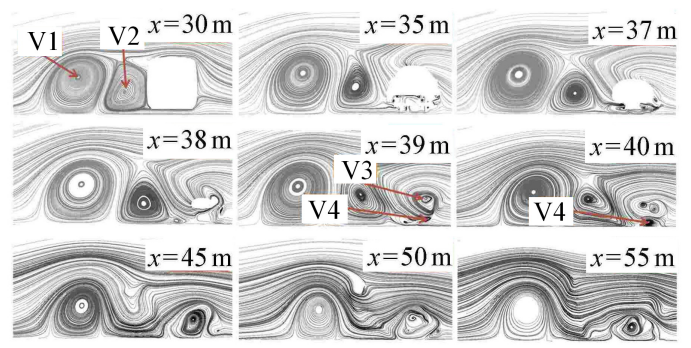

Fig. 18 Streamlines on cross sections at $t=1.5 \mathrm{~s}$

Figure 19 shows the instantaneous streamlines at different time and different cross sections. As can be seen, the intensity and size of V1 change little at $x=30 \mathrm{~m}$ as time goes, but the vortex core of V2 develops slowly at $x=30 \mathrm{~m}$. Since V2 is closer to the train body, its development may affect the aerodynamic force of trailing car more seriously. The small eddies generated around the trailing nose turn to be slightly away from the train. The unsteadiness of the trailing vortices could be obviously observed at $x=50 \mathrm{~m}$. V2 could hardly be detected at $t=0.5 \mathrm{~s}$ while reappears at $t=1 \mathrm{~s}$. Only one vortex core around the trailing nose could 
be found at $t=0.2 \mathrm{~s}$, while another vortex core V6 emerges beneath V 5 at $t=0.5 \mathrm{~s}$. At $t=1 \mathrm{~s}$, a smaller vortex V7 could be observed beneath V6. The vortex intensity and the size of vortex cores vary with time, resulting in time-dependant aerodynamic characteristics for the trailing car.

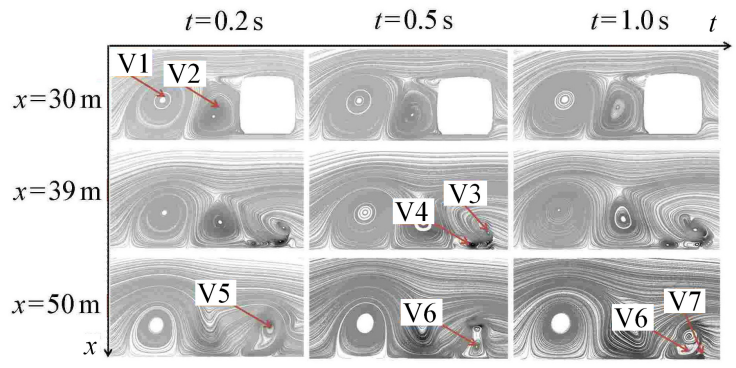

Fig. 19 Instantaneous streamlines at different time and different cross sections

The change of the trailing vortex structure directly affects the aerodynamic force of high speed trains. Figure 20 shows the time histories of $\mathrm{Cl}$ and $\mathrm{Cs}$ of the trailing car in cross wind conditions. It can be seen that the fluctuation of aerodynamic forces of the trailing car change greatly as time elapses. Compared with no cross wind conditions, the lift force and side force of the trailing car increase a lot in cross wind conditions. The lift force increases nearly an order of magnitude, and the side force no longer fluctuations around 0 , but has a large average value, the direction of which points to the leeward side. These arise mainly from the large eddies' influence around the leeward side. A strong low pressure area appears on the leeward side of the train due to V1 and V2. What is more, thanks to the air crash, there is a strong high pressure area on the windward side of the train. As a result, the average values of aerodynamic forces of the trailing car increase a lot. In addition to the continuous generation, development and shedding of small eddies near the trailing cone, the interaction between the small eddies and the two large vortices, make the unsteadiness of the wake flow field more prominent. As can be seen from Fig. 20, at $t=0.2 \mathrm{~s}, 0.5 \mathrm{~s}$, and $1 \mathrm{~s}$, the values of transient aerodynamic forces on the trailing car are quite different. At $t=0.2 \mathrm{~s}$, the small vortices near the trailing cone are not fully developed, so the fluctuation of $C l$ is not serious and the $C l$ is in a valley position. At $t=0.5 \mathrm{~s}$, the small vortices are fully developed, which leads to significantly increase of $C l$. At $t=1 \mathrm{~s}$, thanks to the decrease of intensity of the small vortices around the trailing cone, the fluctuation of $\mathrm{Cl}$ tends to be gentle again. Compared to the small eddies near the trailing car, V1 and V2 have a more serious effect on $C s$. Besides, the development of the two large eddies is very slow, which makes small change in the instantaneous value of $C s$, while the average value of $C s$ varies greatly. At $t=0.5 \mathrm{~s}$, the average value of $C s$ reaches a valley position. However, at $t=1 \mathrm{~s}$, the average value reaches almost a peak position.
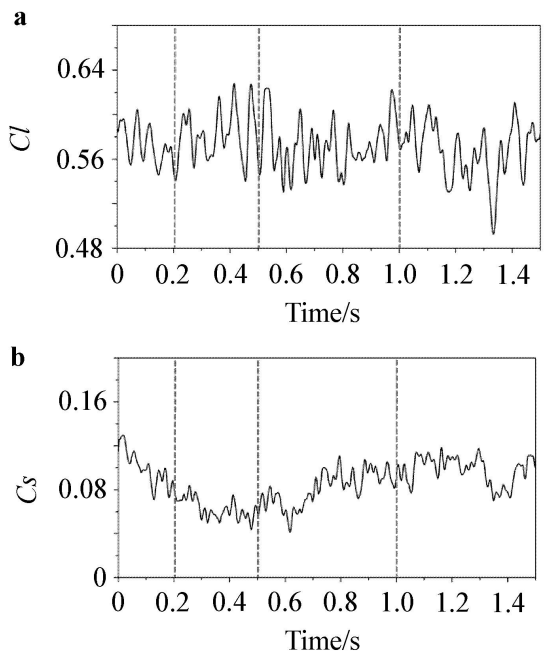

Fig. 20 The time histories of $\mathbf{a} C l$ and $\mathbf{b} C s$ of the trailing car in cross wind conditions

Figure 21 shows the power spectral density of $C l$ and $C s$ in cross wind conditions. As can be seen, the oscillation frequency of $C l$ is $17 \mathrm{~Hz}$, while the value of $C s$ changes to only $1 \mathrm{~Hz}$. The side force of the trailing car is mainly caused by the large eddies on the leeward side, and the development of these large eddies is significantly slower than that of small vortices near the trailing cone, which leads to a small oscillation frequency of $C s$. The mean value of $C l$ is decided by $\mathrm{V} 1$ and V2. The aerodynamic lift on trailing train leeward side is greatly affected by large eddy structures, however, the fluctuation of the instantaneous value is controlled by the small vortices around the trailing car, so the oscillation frequency of $C l$ is obviously larger than that of $C s$.
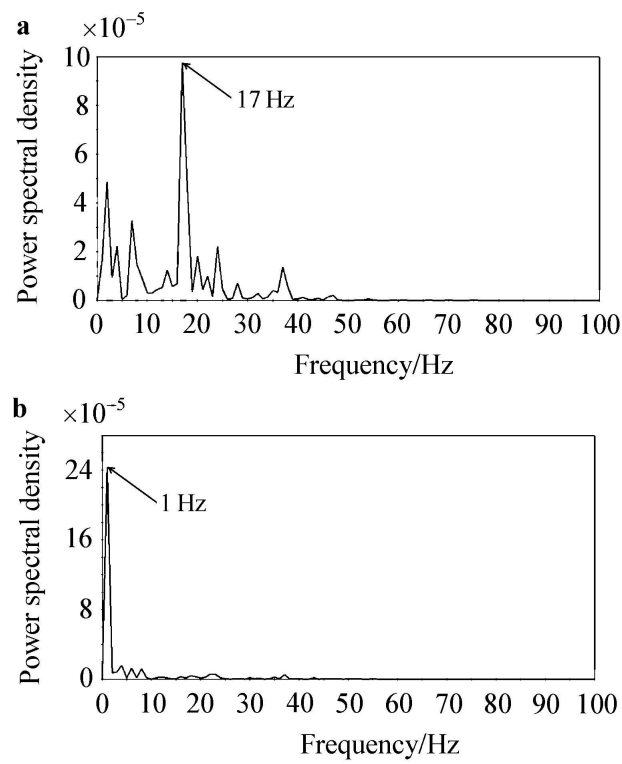

Fig. 21 The power spectral density of $\mathbf{a} C l$ and $\mathbf{b} C s$ in cross wind conditions 
In strong cross wind conditions, a big vortex system generates on the leeward side of the train. Besides, the whole trailing vortex structures have been shifted to the leeward side. As Fig. 22 shows, three big vortices Vc1, Vc2, and $\mathrm{Vc} 4$ compose the main vortex system on the leeward side, which all develop from the leeward side of the leading nose. $\mathrm{Vc} 4$ propagates in a helical mode and disappears in the middle part of the train. An angle of $11.9^{\circ}$ between the vortex core and the train axis has been developed, which is slightly smaller than the yaw angle $\left(13.5^{\circ}\right)$. $\mathrm{Vc} 1, \mathrm{Vc} 2$, and $\mathrm{Vc} 3$ propagate parallel to each other, keeping an angle of $7.4^{\circ}$ with the train axis, which is nearly half of the yaw angle. A smooth vortex core of $\mathrm{Vc} 1$ can be observed and no tiny vortex structures exist around it. Correspondingly, $\mathrm{Vc} 2$ and $\mathrm{Vc} 3$ are both large vortices composed of small vortices. The helical vortices detached from the trailing nose interact with the cross wind, resulting in a complicated oblique vortex Vc3. Meanwhile, small vortices keep generating and collapsing, which would severely disturb the wake flow.

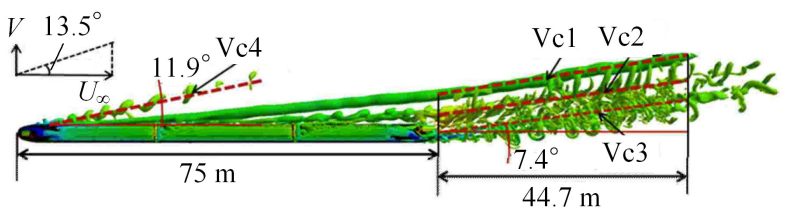

Fig. 22 Surface pressure contour and iso-surface of $Q$ in cross wind conditions $(t=1.5 \mathrm{~s}, Q=100)$

Figure 23 shows instantaneous surface streamlines on the trailing car. It can be observed that four separation lines S1, S2, S3, and S4 exist on the streamlined nose. S4 locates on the leeward side while the other three locate on the windward side. The vortices from S4 together with the upstream vortices are the main sources of Vc2. S2 and S3 combine together, resulting in a strong separation line $S 1$, which is the main source of Vc3. Vortices from S2 and S3 are mostly a result of velocity gradient, and stronger vortices can be observed in S1. Strong vortices collapse into a series of small eddies, which propagate downward and result in the vortex of $\mathrm{Vc} 3$.
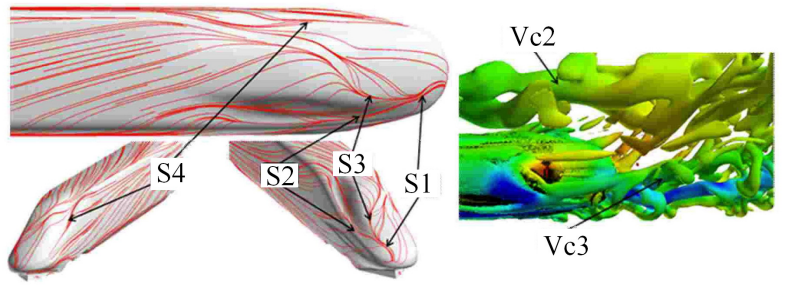

Fig. 23 Surface streamlines and iso-surface of $Q(t=1.5 \mathrm{~s})$

As seen in Fig. 24, there is a big difference in vorticity distribution on planes at different heights. The vortex intensity of $\mathrm{Vc} 4$ is very strong around the leading nose at $z=0 \mathrm{~m}$, while Vc1 begins to develop from the zone around the first bogie. In the zone near the ground, high intensity of $\mathrm{Vc} 2$ and $\mathrm{Vc} 3$ could be observed and combination of these two vortices could also be seen in the wake zone. Vc4 turns to be very weak at $z=1 \mathrm{~m}$. Meanwhile, $\mathrm{Vc1}$ gets a full development and combines with $\mathrm{Vc} 2$ and $\mathrm{Vc} 3$, resulting in a big vortex. Due to the above interaction, small eddies with strong intensity in Vc3 could be clearly detected. On the section of $z=2 \mathrm{~m}, \mathrm{Vc} 4$ almost disappears, and clear boundaries could be observed among $\mathrm{Vc} 1, \mathrm{Vc} 2$, and $\mathrm{Vc} 3$. Vc1 turns to be a big vortex with weak intensity. $\mathrm{Vc} 2$ is composed of small yet long eddies while $\mathrm{Vc} 3$ contains the tiniest and strongest eddies. On the section of $z=3 \mathrm{~m}, \mathrm{Vc} 3$ turns to disappear and the intensity of $\mathrm{Vc} 1$ and $\mathrm{Vc} 2$ becomes even weaker than that at $z=2 \mathrm{~m}$. Meanwhile, the wake zone gets less disturbed than the zone beneath it. In conclusion, Vc1 develops from the first bogie and turns to be a constant yet weak vortex system in the direction away from the ground. The other three vortices, $\mathrm{Vc} 2, \mathrm{Vc} 3$, and $\mathrm{Vc} 4$, interact strongly with the ground. $\mathrm{Vc} 2$ propagates close to the leeward side of the train with strong intensity, which would severely affect the aerodynamic performance. Vc4 disappears before entering the wake zone, indicating that this vortex has no effect on the aerodynamic performance of the trailing car. Vc3 is the main cause that reinforces the turbulence in the wake zone and has strong intensity around the trailing nose, which will heavily worsen the aerodynamic performance of the trailing car. As a result, in cross wind conditions, optimization of the aerodynamic performance of the trailing car should aim at reducing the flow separation around the trailing nose and lowering the intensity of $\mathrm{Vc} 3$.

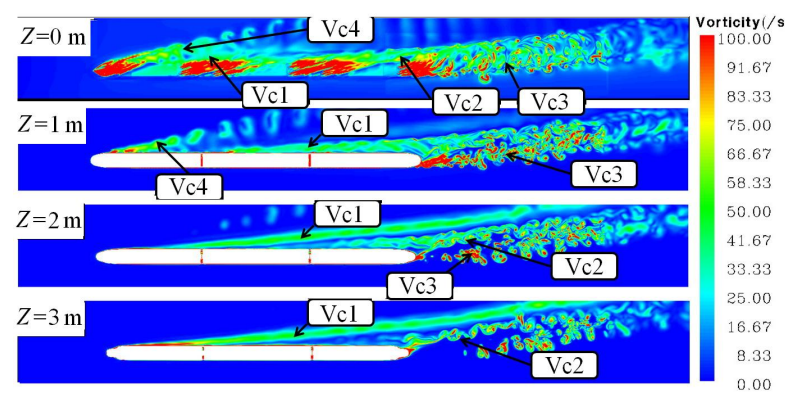

Fig. 24 Instantaneous vorticity contour on different sections $(t=1.5 \mathrm{~s})$

\section{Conclusions}

The wake flow of high speed trains is a typical threedimensional unsteady flow, marked by the generation and collapse of numerous vortices, which would affect severely the aerodynamic performance of the trailing car. Both URANS and DES have been adopted to perform a study on a prototype of $\mathrm{CRH} 2$ high speed trains with three carriages. Several conclusions could be obtained:

(1) DES has a better capacity of capturing small eddies than URANS, but they can both precisely capture the big 
vortex structures. Considering the limitation in mesh amount and computational time, it is more practical to use URANS to perform studies on the evolution of trail vortices.

(2) In conditions without cross wind, two helical vortices exist in the wake zone and interact strongly with the ground. As the velocity increases, the wake zone gets more and more intensive in turbulence. However, the locations where separations take place are not related to the running speed and the height of the trailing vortices is slightly lower than the height of the train.

(3) In cross wind conditions, several vortices exist on the leeward side of the train. Three vortices could be observed in the present cross wind condition. One of the vortices has no effect on the trailing car, while the other two propagate to the wake zone and interact strongly with the helical vortices, resulting in a much more intensive turbulent flow than that in conditions without cross winds. Optimization of the aerodynamic performance of the trailing car should aim at reducing the flow separation around the trailing nose and lowering the intensity of the trail vortex systems.

\section{References}

1 Raghuathan, S., Kim, H.D., Setoguchi, T.: Aerodynamics of high-speed railway train. Progress in Aerospace Sciences 38, 469-514 (2002)

2 Hemida, H., Krajnovic, S.: LES study of the influence of the nose shape and yaw angles on flow structures around trains. J. Wind Eng. Ind. Aerodyn. 98, 34-46 (2010)

3 Baker, C.: The flow around high speed trains. J. Wind Eng. Ind. Aerodyn. 98, 277-298 (2010)

4 Schober, M., Weise, M., Orellano, A., et al.: Wind tunnel investigation of an ICE 3 endcar on thress standard ground scenarios. J. Wind Eng. Ind. Aerodyn. 98, 345-352 (2010)

5 Hemida, H., Baker, C.: Large-eddy simulation of the flow around a freight wagon subjected to a crosswind. Computers \& Fluids 29, 1944-1956 (2010)

6 Wang, Y.W., Wang, Y., An, Y.R., et al.: Aerodynamic simulation of high-speed trains based on the Lattice Boltzmann Method (LBM). Science in China Series E: Technological Sci- ences 51, 773-783 (2008)

7 Yang, Z.G., Ma, J., Chen, Y., et al.: The unsteady aerodynamic characteristics of a high-speed train in different operating conditions under cross wind. Journal of the China Railway Society 32, 18-23 (2010) (in Chinese)

8 Spalart, P.R.: Detached-eddy simulation. Annual Review of Fluid Mechanics 41, 181-202 (2009)

9 Spalart, P.R.: Strategies for turbulence modeling and simulations. Int. J. Heat Fluid Flow 21, 252-263 (2000)

10 Nishino, T., Roberts, G.T., Zhang, X.: Unsteady RANS and detached-eddy simulations of flow around a circular cylinder in ground effect. Journal of Fluids and Structures 24, 18-33 (2008)

11 Hussain, A.K.M.F., Reynolds, W.C.: The mechanics of an organized wave in turbulent shear flow. Journal of Fluid Mechanics 22, 473-537 (1970)

12 Wilcox, D.C.: Turbulence Modeling for CFD. (2nd edn.) DCW Industries, Inc., CA (1998)

13 Caruelle, B., Duckos, F.: Detached-eddy simulations of attached and detached boundary layers. International Journal of Computational Fluid Dynamics 17, 433-451 (2003)

14 Chen, L.W., Xu, C.Y., Lu, X.Y.: Numerical investigation of the compressible flow past an aerofoil. J. Fluid Mech. 643, 97-126 (2010)

15 Kapadia, S., Roy, S., Wurtzler, K.: Detached-eddy simulation over a reference Ahmed car model. In: Proc. of Thermophys. Conf., 36th, Orlando, Paper Number AIAA-0857 (2003)

16 Sreenivas, K., Pankajakshan, R., Nichols, D.S., et al.: Aerodynamic simu-lation of heavy trucks with rotating wheels. In: Proc. of AIAA Aerosp. Sci. Meet. Exhib., 44th, Reno, Pap. No. AIAA-1394 (2006)

17 Blocken, B., Stathopoulos, T., Carmeliet, J.: CFD simulation of the atmospheric boundary layer: wall function problems. Atmospheric Environment 41, 238-252 (2007)

18 Schulte Werning B., Heine, C., Matschke, G.: Unsteady wake characteristics of high speed trains. In: PAMM Proceedings Applied Maths and Mechanics 2, 332-333 (2003)

19 Sima, M., Gurr, A., Orellano, A.: Validation of CFD for the flow under a train with 1:7 scale wind tunnel measurements. BBAA VI International Colloquium on: Bluff bodies aerodynamics and applications. Milano. July, 20-24 (2008)

20 Storms, B.L., Ross, J.C., Heineck, J.T., et al.: An experimental study of the ground transportation system (GTS) model in the NASA Ames 7- by 10-ft wind tunnel. NASA/TM-2001-209621 (2001) 